\title{
The Daugavet equation for polynomials
}

\author{
by \\ Yun Sung Choi (Pohang), Domingo García (Valencia), \\ Manuel Maestre (Valencia) and Miguel Martín (Granada)
}

\begin{abstract}
We study when the Daugavet equation is satisfied for weakly compact polynomials on a Banach space $X$, i.e. when the equality

$$
\|\mathrm{Id}+P\|=1+\|P\|
$$

is satisfied for all weakly compact polynomials $P: X \rightarrow X$. We show that this is the case when $X=C(K)$, the real or complex space of continuous functions on a compact space $K$ without isolated points. We also study the alternative Daugavet equation

$$
\max _{|\omega|=1}\|\operatorname{Id}+\omega P\|=1+\|P\|
$$

for polynomials $P: X \rightarrow X$. We show that this equation holds for every polynomial on the complex space $X=C(K)$ ( $K$ arbitrary) with values in $X$. This result is not true in the real case. Finally, we study the Daugavet and the alternative Daugavet equations for $k$-homogeneous polynomials.
\end{abstract}

In 1963, I. K. Daugavet [13] showed that every compact linear operator $T$ on $C[0,1]$ satisfies

$$
\|\mathrm{Id}+T\|=1+\|T\|,
$$

a norm equality which has become known as the Daugavet equation. Over the years, the validity of the above equality has been established for many classes of operators on many Banach spaces. For instance, weakly compact linear operators on $C(K), K$ perfect, and $L_{1}(\mu), \mu$ atomless, satisfy the Daugavet equation (see [25] for an elementary approach). We refer the reader to the books $[1,2]$ and papers $[20,26]$ for more information and background. It is also a remarkable result given in 1970 by J. Duncan et al. [16] that, for

2000 Mathematics Subject Classification: Primary 46G25; Secondary 46B20, 47A12.

Key words and phrases: polynomial, Banach space, Daugavet equation, numerical range.

The first author was supported by grant No. R01-2004-000-10055-0 from the Basic Research Program of the Korea Science and Engineering Foundation. The second and third authors were supported by MEC and FEDER Project MTM2005-08210. The fourth author was supported by MCYT and FEDER Project BFM2003-01681 and Junta de Andalucía grant FQM-185. 
every compact Hausdorff space $K$ and every bounded linear operator $T$ on $C(K)$, the equality

$$
\max _{\omega \in \mathbb{T}}\|\operatorname{Id}+\omega T\|=1+\|T\|
$$

holds, where we use $\mathbb{T}$ to denote the unit sphere of the base field. The above norm equality is now known as the alternative Daugavet equation [22], and it is satisfied by all bounded linear operators on $C(K)$ and $L_{1}(\mu), K$ and $\mu$ arbitrary. We refer the reader to $[16,21,22]$ and references therein for background. The aim of this paper is to study the Daugavet equation and the alternative Daugavet equation for polynomials in Banach spaces.

There is a concept, the numerical range of an operator (see below for the definition), intimately related to the Daugavet and alternative Daugavet equations. The definition of numerical range for bounded linear operators on Banach spaces was given in 1962 by F. Bauer [5] (see [8, 9] for background) extending the 1918 classical definition of numerical range (or field of values) of a matrix given by O. Toeplitz [24]. In 1968, the concept of numerical range was extended to arbitrary continuous functions from the unit sphere of a real or complex Banach space into the space by F. Bonsall, B. Cain, and H. Schneider [7]. In the seventies, L. Harris [17, 18] showed that a good setting to deal with numerical ranges is the space of bounded uniformly continuous functions on the unit sphere of a Banach space with values in the space (see also Rodríguez-Palacios [23]). Once we give up the linearity, we realize that also the study of the Daugavet and alternative Daugavet equations is clarified if it is done for bounded uniformly continuous functions on the unit ball of a Banach space with values in the space.

Let us introduce the necessary definitions and notations.

Throughout the article, all the Banach spaces considered will be real or complex unless the scalar field is specified, and all polynomials will be continuous. Let $X$ be a Banach space. By $B_{X}$ we denote the closed unit ball and by $S_{X}$ the unit sphere of $X$. Given $k \geq 0$, we denote by $\mathcal{P}\left({ }^{k} X ; X\right)$ the space of all $k$-homogeneous polynomials from $X$ to $X$, and by $\mathcal{P}\left({ }^{k} X\right)$ the space of all $k$-homogeneous scalar polynomials. If $k=0$, we identify $\mathcal{P}\left({ }^{0} X ; X\right)$ with the space of constant functions, i.e. $\mathcal{P}\left({ }^{0} X ; X\right) \equiv X$; for $k=1$, $\mathcal{P}\left({ }^{1} X ; X\right)$ is equal to $L(X)$, the algebra of all bounded linear operators on $X$. We say that $P: X \rightarrow X$ is a polynomial on $X$, and write $P \in \mathcal{P}(X ; X)$, if $P$ is a finite sum of homogeneous polynomials from $X$ to $X$. We use $\mathcal{P}(X)$ to denote the space of all finite sums of homogeneous scalar polynomials. Let us recall that $\mathcal{P}(X ; X)$ is a normed space if we endow it with the norm

$$
\|P\|:=\sup \left\{\|P(x)\|: x \in B_{X}\right\} .
$$

Therefore, $\mathcal{P}(X ; X)$ embeds isometrically into $\ell_{\infty}\left(B_{X}, X\right)$, the Banach space of all bounded functions from $B_{X}$ to $X$ endowed with the supremum norm. 
We will write $\ell_{\infty}\left(B_{X}\right)$ when only scalar-valued functions are considered. Analogous definitions will be used for $B_{X}$ replaced by $S_{X}$.

Generalizing the linear case, we say that $\Phi \in \ell_{\infty}\left(B_{X}, X\right)$ satisfies the Daugavet equation if the norm equality

$$
\|\mathrm{Id}+\Phi\|=1+\|\Phi\|
$$

holds, and we say that $\Phi$ satisfies the alternative Daugavet equation if there exists $\omega \in \mathbb{T}$ such that $\omega \Phi$ satisfies (DE) or, equivalently, if

$$
\max _{|\omega|=1}\|\mathrm{Id}+\omega \Phi\|=1+\|\Phi\| \text {. }
$$

It is clear that (DE) implies (ADE) but, in general, they are not the same (-Id always satisfies (ADE) but never (DE)). Let us mention that a function $\Phi$ satisfies (DE) (resp. (ADE)) if and only if so does $\alpha \Phi$ for every $\alpha \in \mathbb{R}_{0}^{+}$ (see [3, Lemma 2.2], for instance), a fact that we will use in the following without explicit mention. We say that a Banach space $X$ has the $k$-order Daugavet property ( $k$-DP for short) if all rank-one $k$-homogeneous polynomials satisfy (DE). When $k=1$, we simply say that $X$ has the Daugavet property. Analogously, $X$ has the $k$-order alternative Daugavet property $(k$ $A D P$ for short) if all rank-one $k$-homogeneous polynomials satisfy (ADE), and we use the name alternative Daugavet property in the linear case.

If $X$ is a Banach space, we denote by $C_{\mathrm{u}}\left(B_{X}, X\right)$ the Banach space of all uniformly continuous $X$-valued functions on $B_{X}$ endowed with the supremum norm. Note that since $B_{X}$ is convex and bounded, every function in $C_{\mathrm{u}}\left(B_{X}, X\right)$ is also bounded. If $X$ is a complex Banach space, we denote by $\mathcal{A}_{\infty}\left(B_{X}\right)$ (resp. $\left.\mathcal{A}_{\infty}\left(B_{X}, X\right)\right)$ the Banach space of all complex-valued (resp. $X$-valued) functions on $B_{X}$ which are holomorphic in the open unit ball, and bounded and continuous in $B_{X} \cdot \mathcal{A}_{\infty}\left(B_{X}, X\right)$ embeds isometrically into $\ell_{\infty}\left(S_{X}, X\right) . \mathcal{A}_{\mathrm{u}}\left(B_{X}\right)$ (resp. $\mathcal{A}_{\mathrm{u}}\left(B_{X}, X\right)$ ) will stand for the closed subspace of $\mathcal{A}_{\infty}\left(B_{X}\right)$ (resp. $\mathcal{A}_{\infty}\left(B_{X}, X\right)$ ) formed by the functions which admit (a unique) uniformly continuous extension to the closed unit ball of $X$.

For a Banach space $X$, we set

$$
\Pi(X):=\left\{\left(x, x^{*}\right) \in X \times X^{*}: x \in S_{X}, x^{*} \in S_{X^{*}}, x^{*}(x)=1\right\} .
$$

Given a bounded function $\Phi: S_{X} \rightarrow X$, its numerical range is

$$
V(\Phi):=\left\{x^{*}(\Phi(x)):\left(x, x^{*}\right) \in \Pi(X)\right\}
$$

and the associated numerical radius is

$$
v(\Phi):=\sup \{|\lambda|: \lambda \in V(\Phi)\} .
$$

For a bounded function $\Phi: \Omega \rightarrow X$, where $S_{X} \subset \Omega \subset X$, the above definitions apply by just considering $V(\Phi):=V\left(\left.\Phi\right|_{S_{X}}\right)$.

The outline of the paper is as follows. In the first section we give preliminary results about (DE) and (ADE) for arbitrary bounded functions on 
the unit ball of a Banach space with values in the space, analogous to those given in [20, Lemma 2.2 and Theorem 2.3] and [22, Proposition 2.1 and Theorem 2.2] for bounded linear operators. We also relate both equations to numerical ranges in the case of bounded and uniformly continuous functions. We devote Section 2 to (non-homogeneous) polynomials. We prove that every weakly compact polynomial on $C_{\mathrm{b}}(\Omega, X)$ satisfies (DE), where $\Omega$ is a completely regular Hausdorff topological space without isolated points and $X$ is an arbitrary Banach space. We also prove that every polynomial on the complex spaces $c_{0}$ and $C(K)$ ( $K$ arbitrary) satisfies (ADE). Finally, in Section 3 we study the $k$-DP and $k$-ADP. We show that both properties are equivalent in the complex case for $k \geq 2$; the same is true in the real case for $k$ even, but not for $k$ odd. Examples of spaces with the $k$-DP for every $k \geq 2$ are the spaces $C_{\mathrm{b}}(\Omega, X)$ when $\Omega$ has no isolated points, and the complex spaces $c_{0}$ and $C(K)$ ( $K$ arbitrary). The real spaces $c_{0}$ and $C(K)$ when $K$ has isolated points and more than one point do not have the $k$-ADP for every $k \geq 2$. The real or complex space $\ell_{1}$ does not have the $k$-ADP for every $k \geq 2$.

1. Preliminary generalities. Our first preliminary result is a generalization of [20, Lemma 2.2 and Theorem 2.3], where only bounded linear operators were considered. Actually, our proof is inspired by the proof of those results. The following notation will be useful. If $X$ is a Banach space and $\mathcal{Z}$ is a subspace of $\ell_{\infty}\left(B_{X}\right)$, we write $\mathcal{Z}^{X}$ for the space of all functions $\Phi: B_{X} \rightarrow X$ such that $x^{*} \circ \Phi \in \mathcal{Z}$ for every $x^{*} \in X^{*}$. The simplest elements of $\mathcal{Z}^{X}$ are the tensors $\varphi \otimes x_{0}$ for $\varphi \in \mathcal{Z}$ and $x_{0} \in X$ given by $\left[\varphi \otimes x_{0}\right](x)=\varphi(x) x_{0}$ for every $x \in B_{X}$. Let us mention that in this paper we will use the result below for $\mathcal{Z}=\mathcal{P}(X)$ and $\mathcal{Z}=\mathcal{P}\left({ }^{k} X\right)$.

TheOrem 1.1. Let $X$ be a Banach space and let $\mathcal{Z}$ be a subspace of $\ell_{\infty}\left(B_{X}\right)$. Then the following are equivalent:

(i) For every $\varphi \in \mathcal{Z}$ and every $x_{0} \in X, \varphi \otimes x_{0}$ satisfies (DE).

(ii) For every $\varphi \in S_{\mathcal{Z}}$, every $x_{0} \in S_{X}$, and every $\varepsilon>0$, there exist $\omega \in \mathbb{T}$ and $y \in B_{X}$ such that

$$
\operatorname{Re} \omega \varphi(y)>1-\varepsilon \text { and }\left\|x_{0}+\omega y\right\|>2-\varepsilon .
$$

(iii) Every $\Phi \in \mathcal{Z}^{X}$ whose image is relatively weakly compact satisfies (DE).

Proof. (i) $\Rightarrow$ (ii). Let $\Phi=\varphi \otimes x_{0}$; as it satisfies $\|\mathrm{Id}+\Phi\|=2$ by (i), there exists $y \in B_{X}$ such that

$$
\left\|y+\varphi(y) x_{0}\right\|>2-\varepsilon / 2 .
$$


It follows that $|\varphi(y)|>1-\varepsilon / 2$ and, writing $\omega=|\varphi(y)| / \varphi(y) \in \mathbb{T}$, we have

$$
\operatorname{Re} \omega \varphi(y)=|\varphi(y)|>1-\varepsilon / 2>1-\varepsilon
$$

and

$$
\begin{aligned}
\left\|x_{0}+\omega y\right\| & =\left\|y+\bar{\omega} x_{0}\right\|=\left\|y+\frac{\varphi(y)}{|\varphi(y)|} x_{0}\right\| \\
& \geq\left\|y+\varphi(y) x_{0}\right\|-|| \varphi(y)|-1|>2-\varepsilon .
\end{aligned}
$$

(ii) $\Rightarrow$ (iii). Suppose $\|\Phi\|=1$. Since the set $K=\overline{\mathrm{co}}\left(\mathbb{T} \Phi\left(B_{X}\right)\right)$ is weakly compact, it coincides with the closed convex hull of its denting points (see [14] for instance). Therefore, given $\varepsilon>0$, we may take a denting point $y_{0} \in K$ with $\left\|y_{0}\right\|>1-\varepsilon$. Then, for some $0<\delta<\varepsilon$, there is a slice

$$
S=\left\{y \in K: \operatorname{Re} y_{0}^{*}(y) \geq 1-\delta\right\}
$$

of $K$ containing $y_{0}$ and having diameter less than $\varepsilon$; here $y_{0}^{*} \in X^{*}$ and

$$
\sup _{y \in K} \operatorname{Re} y_{0}^{*}(y)=\sup _{y \in K}\left|y_{0}^{*}(y)\right|=1
$$

(observe that $K$ is balanced). In particular,

$$
y \in K, \operatorname{Re} y_{0}^{*}(y)>1-\delta \Rightarrow\left\|y-y_{0}\right\|<\varepsilon .
$$

If we set $\varphi:=y_{0}^{*} \circ \Phi$, then $\varphi \in \mathcal{Z}$ and

$$
\|\varphi\|=\sup _{x \in B_{X}}\left|y_{0}^{*}(\Phi(x))\right|=\sup _{y \in K}\left|y_{0}^{*}(y)\right|=1 .
$$

Now, we use (ii) with $x_{0}=y_{0} /\left\|y_{0}\right\|$ and $\varphi$ to get $y \in B_{X}$ and $\omega \in \mathbb{T}$ such that

$$
\operatorname{Re} \omega \varphi(y)>1-\delta \text { and }\left\|\frac{y_{0}}{\left\|y_{0}\right\|}+\omega y\right\|>2-\delta>2-\varepsilon .
$$

We observe that

$$
\operatorname{Re} y_{0}^{*}(\omega \Phi(y))=\operatorname{Re} \omega \varphi(y)>1-\delta
$$

so (1) and the fact that $\omega \Phi(y) \in K$ give

$$
\left\|\omega \Phi(y)-y_{0}\right\|<\varepsilon .
$$

On the other hand,

$\left\|y_{0}+\omega y\right\| \geq\left\|\omega y+\frac{y_{0}}{\left\|y_{0}\right\|}\right\|-\left\|y_{0}-\frac{y_{0}}{\left\|y_{0}\right\|}\right\|=\left\|\omega y+\frac{y_{0}}{\left\|y_{0}\right\|}\right\|-\left|\left\|y_{0}\right\|-1\right|>2-2 \varepsilon$.

Finally,

$\|\operatorname{Id}+\Phi\| \geq\|y+\Phi(y)\|=\|\omega(y+\Phi(y))\| \geq\left\|\omega y+y_{0}\right\|-\left\|\omega \Phi(y)-y_{0}\right\|>2-3 \varepsilon$.

Letting $\varepsilon \downarrow 0$, we conclude that $\Phi$ satisfies (DE).

(iii) $\Rightarrow$ (i) is clear.

For the alternative Daugavet equation we can state an analogous result, as in [22, Proposition 2.1 and Theorem 2.2] for the linear case. Part of it is 
a direct consequence of the above theorem, and the proof of the other part can be easily adapted.

Corollary 1.2. Let $X$ be a Banach space and let $\mathcal{Z}$ be a subspace of $\ell_{\infty}\left(B_{X}\right)$. Then the following are equivalent:

(i) For every $\varphi \in \mathcal{Z}$ and every $x_{0} \in X, \varphi \otimes x_{0}$ satisfies (ADE).

(ii) For every $\varphi \in S_{\mathcal{Z}}$, every $x_{0} \in S_{X}$, and every $\varepsilon>0$, there exist $\omega_{1}, \omega_{2} \in \mathbb{T}$ and $y \in B_{X}$ such that

$$
\operatorname{Re} \omega_{1} \varphi(y)>1-\varepsilon \text { and }\left\|x_{0}+\omega_{2} y\right\|>2-\varepsilon .
$$

(iii) For every $\varphi \in S_{\mathcal{Z}}$, every $x_{0} \in S_{X}$, and every $\varepsilon>0$, there exist $\omega \in \mathbb{T}$ and $y \in B_{X}$ such that

$$
|\varphi(y)|>1-\varepsilon \text { and }\left\|x_{0}+\omega y\right\|>2-\varepsilon .
$$

(iv) Every $\Phi \in \mathcal{Z}^{X}$ whose image is relatively weakly compact satisfies (ADE).

Proof. The equivalence between (i) and (ii) follows from Theorem 1.1 and the fact that $\varphi \otimes x_{0}$ satisfies (ADE) if and only if there exists $\omega \in \mathbb{T}$ such that $\omega \varphi \otimes x_{0}$ satisfies (DE). (ii) and (iii) are trivially equivalent, and (iv) $\Rightarrow$ (i) is clear. The proof of (ii) $\Rightarrow$ (iv) is a straightforward adaptation of the argument in the proof of Theorem 1.1.

For uniformly continuous functions we can state other characterizations of the equations (DE) and (ADE). The proof can be deduced from the general theory of numerical ranges [18], by just proving that the norm of a uniformly continuous function satisfying (DE) can be calculated using only elements of the unit sphere of the space. At the same price, we will give a direct proof.

Proposition 1.3. Let $X$ be a Banach space and let $\Phi$ be an element of $C_{\mathrm{u}}\left(B_{X}, X\right)$. Then:

(a) $\Phi$ satisfies (DE) if and only if $\|\Phi\|=\sup \operatorname{Re} V(\Phi)$.

(b) $\Phi$ satisfies (ADE) if and only if $\|\Phi\|=v(\Phi)$.

Proof. (a) Suppose that $\Phi$ satisfies (DE). Since $\Phi$ is uniformly continuous, for every $\varepsilon>0$ there exists $0<\delta<\varepsilon$ such that

$$
y, z \in B_{X},\|y-z\|<\delta \Rightarrow\|\Phi(y)-\Phi(z)\|<\varepsilon .
$$

For every fixed $0<\varepsilon<1$, we may find $y \in B_{X}$ such that $\|y+\Phi(y)\|>$ $1+\|\Phi\|-\delta^{2} / 4$, and then we may also find $y^{*} \in S_{X^{*}}$ such that

$$
\operatorname{Re} y^{*}(y)+\operatorname{Re} y^{*}(\Phi(y))>1+\|\Phi\|-\delta^{2} / 4 .
$$


It clearly follows that

$$
\begin{gathered}
\operatorname{Re} y^{*}(y)>1-\delta^{2} / 4 \\
\operatorname{Re} y^{*}(\Phi(y))>\|\Phi\|-\delta^{2} / 4>\|\Phi\|-\varepsilon .
\end{gathered}
$$

By the Bishop-Phelps-Bollobás theorem [9, §16], we deduce from (2) the existence of a pair $\left(z, z^{*}\right) \in \Pi(X)$ such that

$$
\|y-z\|<\delta \text { and }\left\|y^{*}-z^{*}\right\|<\delta<\varepsilon .
$$

Now, we have $\|\Phi(y)-\Phi(z)\|<\varepsilon$ by the uniform continuity of $\Phi$ and so

$$
\begin{aligned}
& \left|\operatorname{Re} z^{*}(\Phi(z))-\operatorname{Re} y^{*}(\Phi(y))\right| \\
& \quad \leq\left|\operatorname{Re} z^{*}(\Phi(z)-\Phi(y))\right|+\left|\operatorname{Re}\left[y^{*}-z^{*}\right](\Phi(z))\right|+\left|\operatorname{Re} y^{*}(\Phi(y)-\Phi(z))\right| \\
& \leq\|\Phi(z)-\Phi(y)\|+\left\|y^{*}-z^{*}\right\|+\|\Phi(y)-\Phi(z)\|<3 \varepsilon
\end{aligned}
$$

From this and (3) we deduce that

$$
\operatorname{Re} z^{*}(\Phi(z))>\|\Phi\|-4 \varepsilon .
$$

Let us prove the converse implication. For every $\varepsilon>0$, we may find a pair $\left(z, z^{*}\right) \in \Pi(X)$ such that

Therefore,

$$
\operatorname{Re} z^{*}(\Phi(z))>\|\Phi\|-\varepsilon
$$

$$
\|\operatorname{Id}+\Phi\| \geq \operatorname{Re} z^{*}(z+\Phi(z))=1+\operatorname{Re} z^{*}(\Phi(z))>1+\|\Phi\|-\varepsilon,
$$

and the result follows by letting $\varepsilon \downarrow 0$.

(b) If $\Phi$ satisfies (ADE), then there exists $\omega \in \mathbb{T}$ such that $\omega \Phi$ satisfies (DE) and, by (a), we have

$$
\|\Phi\|=\|\omega \Phi\|=\sup \operatorname{Re} V(\omega \Phi) \leq v(\Phi) \leq\|\Phi\| .
$$

Conversely, given $\left(x, x^{*}\right) \in \Pi(X)$, we have

$$
\max _{\omega \in \mathbb{T}}\|\operatorname{Id}+\omega \Phi\| \geq \max _{\omega \in \mathbb{T}}\left|x^{*}(x)+\omega x^{*}(\Phi(x))\right|=1+\left|x^{*}(\Phi(x))\right| .
$$

Therefore,

$$
\max _{\omega \in \mathbb{T}}\|\operatorname{Id}+\omega \Phi\| \geq 1+\sup \left\{\left|x^{*}(\Phi(x))\right|:\left(x, x^{*}\right) \in \Pi(X)\right\}=1+\|\Phi\|,
$$

and the result follows since the other inequality is always true.

Remark 1.4. Let $X$ be a Banach space and $\Phi \in C_{\mathrm{u}}\left(B_{X}, X\right)$. As a consequence of the above proposition, if $\Phi$ satisfies (ADE), then the norm of $\Phi$ can be calculated using only elements in $S_{X}$, that is, $\|\Phi\|=\sup \{\|\Phi(y)\|$ : $\left.y \in S_{X}\right\}$.

2. The Daugavet equation for polynomials. We start the section by studying the simplest examples: $\mathbb{R}$ and $\mathbb{C}$. 
EXAMPLES 2.1.

(a) Since the linear operators on a Banach space are polynomials, neither $\mathbb{R}$ nor $\mathbb{C}$ has the property that every (weakly compact) polynomial on them satisfies (DE).

(b) Every polynomial on $\mathbb{C}$ satisfies (ADE). Indeed, fix $P \in \mathcal{P}(\mathbb{C} ; \mathbb{C})$ with $\|P\|=1$. Then, by the maximum modulus theorem, there exists $y \in S_{\mathbb{C}} \equiv \mathbb{T}$ such that $|P(y)|=\|P\|=1$, and we may find $\omega_{1} \in \mathbb{T}$ such that

$$
\operatorname{Re} \omega_{1} P(y)=|P(y)|=1 \text {. }
$$

On the other hand, we may find $\omega_{2} \in \mathbb{T}$ such that

$$
\operatorname{Re} \omega_{2} y=|y|=1 \text {. }
$$

Then, writing $\omega=\bar{\omega}_{2} \omega_{1} \in \mathbb{T}$, we have

$$
\|\mathrm{Id}+\omega P\| \geq|y+\omega P(y)|=\left|\omega_{2} y+\omega_{1} P(y)\right|=2 .
$$

(c) The above result is not valid in the real case, i.e. there exists a (weakly compact) polynomial $P \in \mathcal{P}(\mathbb{R} ; \mathbb{R})$ such that $\|\mathrm{Id} \pm P\|<$ $1+\|P\|$. Indeed, if we define

$$
P(t)=1-t^{2} \quad(t \in \mathbb{R}),
$$

we have $\|P\|=1$ and

$$
\|\mathrm{Id} \pm P\|=\max _{t \in[-1,1]}\left|t \pm\left(1-t^{2}\right)\right|=5 / 4<2 .
$$

Our next aim is to present a wide family of Banach spaces in which all weakly compact polynomials satisfy (DE). We will use Theorem 1.1, which in terms of polynomials reads as follows.

Corollary 2.2. Let $X$ be a Banach space. Then the following are equivalent:

(i) For every $p \in \mathcal{P}(X)$ and every $x_{0} \in X$, the polynomial $p \otimes x_{0}$ satisfies (DE).

(ii) For every $p \in \mathcal{P}(X)$ with $\|p\|=1$, every $x_{0} \in S_{X}$, and every $\varepsilon>0$, there exist $\omega \in \mathbb{T}$ and $y \in B_{X}$ such that

$$
\operatorname{Re} \omega p(y)>1-\varepsilon \quad \text { and } \quad\left\|x_{0}+\omega y\right\|>2-\varepsilon \text {. }
$$

(iii) Every weakly compact $P \in \mathcal{P}(X ; X)$ satisfies (DE).

Definition 2.3. Let $\Omega$ be a completely regular Hausdorff topological space and let $X$ be a Banach space. We say that a subspace $\mathcal{F}$ of $C_{\mathrm{b}}(\Omega, X)$, the Banach space of all bounded $X$-valued continuous functions on $\Omega$ endowed with the supremum norm, is $C_{\mathrm{b}}$-rich if for every open subset $U$ of $\Omega$, every $x \in X$ and every $\varepsilon>0$ there exists a continuous function $\varphi: \Omega \rightarrow[0,1]$ of norm one with support included in $U$ such that the distance of $\varphi \otimes x$ to $\mathcal{F}$ is less than $\varepsilon$. 
We can assume in the definition that there is $t_{0} \in U$ such that $\varphi\left(t_{0}\right)=1$. Indeed, given $0<\varepsilon<1$, consider $\varphi$ as above. Since $\varphi$ has norm one and support in $U$, we can find $t_{0} \in U$ and an open neighborhood $V$ of $t_{0}$ such that $\varphi(t)>1-\varepsilon$ for all $t \in V$. As $\Omega$ is completely regular, there exists $\eta: \Omega \rightarrow[0,1]$ such that $\eta\left(t_{0}\right)=1$ and $\eta$ vanishes in $\Omega \backslash V$. If we set $\phi(t)=\max \{\varphi(t), \eta(t)\}$ for $t \in \Omega$ then $\phi: \Omega \rightarrow[0,1]$ is continuous, it has support included in $U, \phi\left(t_{0}\right)=1$ and $\|\varphi-\phi\| \leq \varepsilon$, thus $\operatorname{dist}(\phi \otimes x, \mathcal{F})<2 \varepsilon$.

This definition is a straightforward extension of the definition of $C$-rich subspace of $C(K)$ given in [10, Definition 2.3].

Now, we can state the main result of the section.

THEOREM 2.4. Let $\Omega$ be a completely regular Hausdorff topological space without isolated points, let $X$ be a Banach space and let $\mathcal{F}$ be a $C_{\mathrm{b}}$-rich subspace of $C_{\mathrm{b}}(\Omega, X)$. Then every weakly compact polynomial from $\mathcal{F}$ into itself satisfies the Daugavet equation.

Proof. Let $p \in \mathcal{P}(\mathcal{F})$ with $\|p\|=1$ and fix $f_{0} \in S_{\mathcal{F}}$. Since $p$ is uniformly continuous when restricted to any bounded subset of $\mathcal{F}$, given $\varepsilon>0$, there exists $0<\delta<\min \{\varepsilon / 10,1 / 6\}$ such that

$$
|p(f)-p(g)|<\varepsilon / 2
$$

for all $f, g \in \mathcal{F}$ satisfying $\|f-g\|<2 \delta,\|f\| \leq 2,\|g\| \leq 2$. We take $h \in \mathcal{F}$ with $\|h\| \leq 1$ such that $|p(h)|>1-\varepsilon / 4$, and also $\omega \in \mathbb{T}$ such that

$$
\operatorname{Re} \omega p(h)=|p(h)|>1-\varepsilon / 4 .
$$

Fix a point $\tau \in \Omega$ with $\left\|f_{0}(\tau)\right\|>1-\delta / 4$ and find an open neighborhood $U$ of $\tau$ such that

$$
\left\|f_{0}(t)-f_{0}(\tau)\right\|<\delta / 4
$$

for all $t \in U$. Decreasing $U$ if necessary, we may assume that

$$
\|h(s)-h(t)\|<\delta / 2
$$

for all $s, t \in U$. Select a sequence $U_{1}, U_{2}, \ldots$ of non-empty pairwise disjoint open subsets of $U$. We fix $s_{j} \in U_{j}$ for all $j=1,2, \ldots$ and define $x_{j}=$ $\omega^{-1} f_{0}\left(s_{j}\right)-h\left(s_{j}\right)$. As $\mathcal{F}$ is $C_{\mathrm{b}}$-rich, we can find continuous non-negative functions $\eta_{j}: \Omega \rightarrow[0,1]$ of norm one and $z_{j} \in \mathcal{F}$ such that

$$
\operatorname{supp} \eta_{j} \subset U_{j} \quad \text { and } \quad\left\|\eta_{j} \otimes x_{j}-z_{j}\right\|<\frac{\delta}{2^{j+2}}\left\|\eta_{j} \otimes x_{j}\right\|
$$

for all $j \in \mathbb{N}$. By disjointness of their supports the functions $\eta_{j} \otimes x_{j} /\left\|\eta_{j} \otimes x_{j}\right\|$ form a sequence 1-equivalent to the canonical basis of $c_{0}$. Thanks to (6), the sequence $\left(z_{j}\right)$ spans a subspace isomorphic to $c_{0}$ and tends weakly to zero. So by the Bogdanowicz theorem on weak continuity of polynomials on $c_{0}$ ([6], see also [15, Proposition 1.59]), $\left|p\left(h+z_{j}\right)-p(h)\right|$ tends to 0 as $j \rightarrow \infty$. 
So there is a $j_{0}$ such that

$$
\left|p\left(h+z_{j_{0}}\right)-p(h)\right|<\varepsilon / 4 .
$$

Combining with (5), we have

$$
\operatorname{Re} \omega p\left(h+z_{j_{0}}\right)>1-\varepsilon / 2 .
$$

We define

$$
A=h+z_{j_{0}}, \quad B=h+\eta_{j} \otimes x_{j}, \quad g=A /\|A\| .
$$

Clearly, $B \in C_{\mathrm{b}}(\Omega, X), A, g \in \mathcal{F},\|g\|=1$, and $\|A-B\|<\delta / 2$. We have

$$
1+2 \delta>\|A\|>1-2 \delta .
$$

Indeed, for the lower bound, select $t_{0} \in U_{j_{0}}$ for which $\eta_{j_{0}}\left(t_{j_{0}}\right)=1$; then

$$
\begin{aligned}
\|A\| & \geq\left\|B\left(t_{j_{0}}\right)\right\|-\|A-B\| \\
& =\left\|h\left(t_{j_{0}}\right)-h\left(s_{j_{0}}\right)+\omega^{-1} f_{0}\left(s_{j_{0}}\right)\right\|-\|A-B\| \\
& \geq\left\|f_{0}\left(s_{j_{0}}\right)\right\|-\left\|h\left(t_{j_{0}}\right)-h\left(s_{j_{0}}\right)\right\|-\delta / 2>1-2 \delta .
\end{aligned}
$$

On the other hand, for the upper bound,

$$
\|A\| \leq\|B\|+\|A-B\|<\|B\|+\delta / 2
$$

and $\|B(t)\|=\|h(t)\| \leq 1$ if $t \notin U_{j_{0}}$; if $t \in U_{j_{0}}$ then

$$
\|B(t)\| \leq\left\|h(t)-h\left(s_{j_{0}}\right)\right\|+\left(1-\eta_{j_{0}}(t)\right)\left\|h\left(s_{j_{0}}\right)\right\|+\eta_{j_{0}}(t)\left\|f_{0}\left(s_{j_{0}}\right)\right\|<1+\delta .
$$

Now, by (8),

$$
\|g-A\|=|1-\|A\||<2 \delta .
$$

Hence, by (4) and (7),

$$
\operatorname{Re} \omega p(g)>1-\varepsilon,
$$

and

$$
\begin{aligned}
\left\|f_{0}+\omega g\right\| \geq & \left\|f_{0}+\omega \frac{B}{\|A\|}\right\|-\frac{\|A-B\|}{\|A\|} \\
\geq & \left\|f_{0}\left(t_{j_{0}}\right)+\omega \frac{B\left(t_{j_{0}}\right)}{\|A\|}\right\|-\frac{\delta}{2\|A\|} \\
\geq & \left\|f_{0}\left(t_{j_{0}}\right)+\frac{\omega\left(h\left(t_{j_{0}}\right)-h\left(s_{j_{0}}\right)\right)+f_{0}\left(s_{j_{0}}\right)}{\|A\|}\right\|-\frac{\delta}{2\|A\|} \\
\geq & \left\|f_{0}\left(s_{j_{0}}\right)+\frac{f_{0}\left(s_{j_{0}}\right)}{\|A\|}\right\|-\left\|f_{0}\left(t_{j_{0}}\right)-f_{0}\left(s_{j_{0}}\right)\right\| \\
& -\frac{\left\|h\left(t_{j_{0}}\right)-h\left(s_{j_{0}}\right)\right\|}{\|A\|}-\frac{\delta}{2\|A\|} \\
> & \left\|f_{0}\left(s_{j_{0}}\right)\right\|\left(1+\frac{1}{\|A\|}\right)-\frac{\delta}{2}-\frac{\delta}{\|A\|}-\frac{\delta}{2\|A\|}>2-\varepsilon .
\end{aligned}
$$


The last inequality is a consequence of the fact that $\left\|f_{0}\left(s_{j_{0}}\right)\right\|>1-\delta / 2$, of (8), and of the choice of $\delta$. By Corollary 2.2, every weakly compact polynomial from $\mathcal{F}$ into itself satisfies the Daugavet equation.

If $\Omega$ is a completely regular Hausdorff topological space and $X$ is a Banach space, then $C_{\mathrm{b}}(\Omega, X)$ is $C_{\mathrm{b}}$-rich in itself. Hence we have the following straightforward corollary.

COROLlary 2.5. Let $\Omega$ be a completely regular Hausdorff topological space without isolated points and let $X$ be a Banach space. Then every weakly compact polynomial from $C_{\mathrm{b}}(\Omega, X)$ to itself satisfies the Daugavet equation.

The density of the space of polynomials on a complex Banach space $Z$ in $\mathcal{A}_{\mathrm{u}}\left(B_{Z}, Z\right)$ gives us the following consequence of the above corollary.

COROLlary 2.6. Let $\Omega$ be a completely regular Hausdorff topological space without isolated points and let $X$ be a complex Banach space. Then every weakly compact $\Phi$ in $\mathcal{A}_{\mathrm{u}}\left(B_{C_{\mathrm{b}}(\Omega, X)}, C_{\mathrm{b}}(\Omega, X)\right)$ satisfies the Daugavet equation.

Proof. We denote by $P_{k}$ the $k$-homogeneous polynomial of the Taylor series expansion of $\Phi$ at 0 . By the Cauchy integral formula, $P_{k}\left(B_{X}\right)$ is contained in the closed and absolutely convex hull of $\Phi\left(B_{X}\right)$. As a consequence, $P_{k}$ is weakly compact for all $k$. Hence, the Taylor polynomials of $\Phi$ are also weakly compact. For each $n, \Phi_{n}(f):=\Phi\left(\frac{n-1}{n} f\right)$ belongs to $\mathcal{A}_{\mathrm{u}}\left(B_{C_{\mathrm{b}}(\Omega, X)}, C_{\mathrm{b}}(\Omega, X)\right)$ and the sequence $\left(\Phi_{n}\right)$ converges uniformly to $\Phi$ on the closed unit ball of $C_{\mathrm{b}}(\Omega, X)$. Moreover, for each $\Phi_{n}$ its Taylor series expansion converges again uniformly to $\Phi_{n}$ on the closed unit ball of $C_{\mathrm{b}}(\Omega, X)$. Thus, given $\varepsilon>0$ there exists a weakly compact polynomial $P$ such that

$$
\|\Phi-P\|<\varepsilon .
$$

Hence, by Theorem 2.4, we have

$$
\begin{aligned}
\|\operatorname{Id}+\Phi\| & \geq\|\operatorname{Id}+P\|-\|\Phi-P\|=1+\|P\|-\|\Phi-P\| \\
& \geq 1+\|\Phi\|-2\|\Phi-P\|>1+\|\Phi\|-2 \varepsilon,
\end{aligned}
$$

and the conclusion follows.

More examples of $C_{\mathrm{b}}$-rich subspaces of $C_{\mathrm{b}}(\Omega, X)$ spaces appear in [19] (see [10, Proposition 2.5]): if $K$ is a compact space without isolated points, any finite-codimensional subspace of $C(K)$ is C-rich in $C(K)$, and Theorem 2.4 applies. Therefore, we get the following.

Corollary 2.7. Let $K$ be a compact Hausdorff topological space without isolated points, and let $Y$ be a finite-codimensional subspace of $C(K)$. Then every weakly compact polynomial in $\mathcal{P}(Y ; Y)$ satisfies $(\mathrm{DE})$. 
If $K$ is a compact Hausdorff topological space with isolated points, the above result is not valid, since there exist weakly compact polynomials from $C(K)$ into itself which do not satisfy (DE) (actually, the examples can be operators). One may wonder if at least every weakly compact polynomial from $C(K)$ into itself satisfies (ADE). In the real case, this is not the case, since we have seen at the beginning of this section (Example 2.1(c)) that even in the simplest case in which $K$ has only one element (i.e. $C(K)=\mathbb{R}$ ), there are (weakly compact) polynomials which do not satisfy (ADE). In the complex case, the situation is completely different, as we see in the next result.

TheOREm 2.8. Let $K$ be a compact Hausdorff space and let $X$ be the complex space $C(K)$. Then $v(\Phi)=\|\Phi\|$ for every $\Phi \in \mathcal{A}_{\infty}\left(B_{X}, X\right)$.

To prove the above theorem, we need a preliminary result.

Lemma 2.9. Let $\Omega$ be a set, let $\mathcal{F}$ be a subspace of $\ell_{\infty}(\Omega)$, and let $\Lambda \subseteq \Omega$ be a norming set for $\mathcal{F}$ (i.e. $\|f\|=\sup \{|f(\lambda)|: \lambda \in \Lambda\}$ for every $f \in \mathcal{F})$. Then, given a Banach space $Y$ and a function $\Phi \in \ell_{\infty}(\Omega, Y)$ such that $y^{*} \circ \Phi \in \mathcal{F}$ for every $y^{*} \in Y^{*}$, we have

$$
\|\Phi\|=\sup \{\|\Phi(\lambda)\|: \lambda \in \Lambda\} .
$$

Proof. We clearly have

$$
\begin{aligned}
\|\Phi\| & =\sup _{t \in \Omega}\|\Phi(t)\|=\sup _{t \in \Omega} \sup _{y^{*} \in B_{Y^{*}}}\left|y^{*}(\Phi(t))\right| \\
& =\sup _{y^{*} \in B_{Y^{*}}} \sup _{t \in \Omega}\left|y^{*}(\Phi(t))\right|=\sup _{y^{*} \in B_{Y^{*}}}\left\|y^{*} \circ \Phi\right\| \\
& =\sup _{y^{*} \in B_{Y^{*}}} \sup _{t \in \Lambda}\left|\left[y^{*} \circ \Phi\right](t)\right|=\sup _{t \in \Lambda} \sup _{y^{*} \in B_{Y^{*}}}\left|\left[y^{*} \circ \Phi\right](t)\right|=\sup _{t \in \Lambda}\|\Phi(t)\| .
\end{aligned}
$$

Proof of Theorem 2.8. By [4, Theorem 4.3], the set $\operatorname{Ext}\left(B_{C(K)}\right)$ of all extreme points of $B_{C(K)}$ is a norming set for $A_{\infty}\left(B_{C(K)}\right)$ and, by the above lemma, given $\varepsilon>0$, there exists $e \in \operatorname{Ext}\left(B_{C(K)}\right)$ such that $\|\Phi(e)\|>\|\Phi\|-\varepsilon$. On one hand, since $\Phi(e) \in C(K)$, there exists $t \in K$ such that $[\Phi(e)](t)=$ $\|\Phi(e)\|$. On the other hand, since $e \in \operatorname{Ext}\left(B_{C(K)}\right)$, we have $|e(t)|=1$. Now, we observe that

$$
\left|\delta_{t}(\Phi(e))\right|=|[\Phi(e)](t)|=\|\Phi(e)\|>\|\Phi\|-\varepsilon,
$$

and $\left|\delta_{t}(e)\right|=|e(t)|=1$. Therefore, $v(\Phi) \geq\|\Phi\|-\varepsilon$.

In particular,

Corollary 2.10. Let $K$ be a compact Hausdorff space. Then every polynomial from the complex space $C(K)$ into itself satisfies (ADE). 
EXAmple 2.11. Let $X$ be the complex space $c_{0}$. Then every polynomial from $X$ into $X$ satisfies (ADE). Indeed, given $\Phi \in \mathcal{A}_{\mathrm{u}}\left(B_{X}, X\right)$ and $\varepsilon>0$, there exists $x_{0} \in S_{X}$ such that $\left\|\Phi\left(x_{0}\right)\right\|>\|\Phi\|-\varepsilon$. Thus, we can find $j$ such that

$$
\left|e_{j}^{*}\left(\Phi\left(x_{0}\right)\right)\right|>\|\Phi\|-\varepsilon,
$$

where $e_{j}^{*}$ is the functional associated to the $j$ th element $e_{j}$ of the canonical basis of $X$. We define $f: \mathbb{C} \rightarrow \mathbb{C}$ by

$$
f(z)=e_{j}^{*}\left(\Phi\left(x_{0}+\left(z-e_{j}^{*}\left(x_{0}\right)\right) e_{j}\right)\right) \quad(z \in \mathbb{C}) .
$$

It is an element of $\mathcal{A}_{\mathrm{u}}\left(B_{\mathbb{C}}\right)$. By the maximum modulus theorem, there exists $z_{0} \in \mathbb{C}$ with $\left|z_{0}\right|=1$ such that $|f(z)| \leq\left|f\left(z_{0}\right)\right|$ for all $z$ in the closed unit disk of $\mathbb{C}$. In particular,

$\|\Phi\|-\varepsilon<\left|e_{j}^{*}\left(\Phi\left(x_{0}\right)\right)\right|=\left|f\left(e_{j}^{*}\left(x_{0}\right)\right)\right| \leq\left|f\left(z_{0}\right)\right|=\left|e_{j}^{*}\left(\Phi\left(x_{0}+\left(z_{0}-e_{j}^{*}\left(x_{0}\right)\right) e_{j}\right)\right)\right|$.

But $x_{1}:=x_{0}+\left(z_{0}-e_{j}^{*}\left(x_{0}\right)\right) e_{j} \in S_{X}$, and clearly $\left|e_{j}^{*}\left(x_{1}\right)\right|=1$. Hence,

$$
\|\Phi\|-\varepsilon<\left|e_{j}^{*}\left(\Phi\left(x_{1}\right)\right)\right| \leq v(\Phi) .
$$

Finally, Proposition 1.3 yields the conclusion.

In [12, Theorem 3.1] it is proved that every continuous $k$-linear mapping $A: X^{k} \rightarrow X$ satisfies $v(A)=\|A\|$ for $X$ being $c_{0}, c$ and $\ell_{\infty}$, and it is claimed that every $k$-homogeneous polynomial $P$ on $X$ satisfies $v(P)=\|P\|$. Unfortunately the last claim is false for the cases of real $c_{0}, c$ and $\ell_{\infty}$ as we will show in Example 3.14. Nevertheless, the claim is true for the complex $c_{0}, c$ and $\ell_{\infty}$, not only for $k$-homogeneous polynomials but for any element of $\mathcal{A}_{\mathrm{u}}\left(B_{X}, X\right)$, as we have shown in Theorem 2.8 and in the proof of Example 2.11.

3. The Daugavet equation for $k$-homogeneous polynomials. The aim of this section is to study the $k$-order Daugavet property and the $k$-order alternative Daugavet property. This study makes sense since Example 3.4(b) below shows that there exist spaces with weakly compact polynomials which do not satisfy (ADE) and nevertheless every weakly compact $k$-homogeneous polynomial satisfies (ADE).

We start by presenting the first examples of spaces with the $k$-DP and $k$-ADP. The results are obvious consequences of Theorem 2.4, Corollary 2.10, and Example 2.11.

EXAMPLES 3.1.

(a) Let $\Omega$ be a completely regular Hausdorff topological space without isolated points, and let $X$ be a Banach space. Then the space $C_{\mathrm{b}}(\Omega, X)$ has the $k$-order Daugavet property for every $k \in \mathbb{N}$.

(b) Let $K$ be a compact Hausdorff space. Then the complex space $C(K)$ has the $k$-order alternative Daugavet property for every $k \in \mathbb{N}$. 
(c) The complex space $c_{0}$ has the $k$-order alternative Daugavet property for every $k \in \mathbb{N}$.

The following result shows a surprising behavior of the Daugavet equation and the alternative Daugavet equation for $k$-homogeneous polynomials with $k>1$.

Proposition 3.2. Let $X$ be a Banach space over $\mathbb{K}$ and let $k$ be an integer, $k \geq 2$.

(a) If $\mathbb{K}=\mathbb{C}$, then (DE) and ( $\mathrm{ADE})$ are equivalent in $\mathcal{P}\left({ }^{k} X ; X\right)$.

(b) If $\mathbb{K}=\mathbb{R}$ and $k$ is even, then (DE) and ( $\mathrm{ADE}$ ) are equivalent in $\mathcal{P}\left({ }^{k} X ; X\right)$.

Proof. Only $(\mathrm{ADE}) \Rightarrow(\mathrm{DE})$ has to be proved.

(a) If $P \in \mathcal{P}\left({ }^{k} X ; X\right)$ satisfies (ADE), then there is $\omega \in \mathbb{T}$ such that

$$
\|\mathrm{Id}+\omega P\|=1+\|P\| .
$$

We take $\beta \in \mathbb{T}$ such that $\beta^{k-1}=\omega$ (observe that we need $k \geq 2$ for this!). For every $\varepsilon>0$, we may find $x \in B_{X}$ such that

$$
\|x+\omega P(x)\|>1+\|P\|-\varepsilon .
$$

If we write $y=\beta x \in B_{X}$, we have

$$
\begin{aligned}
\|y+P(y)\| & =\|\beta x+P(\beta x)\|=\left\|\beta x+\beta^{k} P(x)\right\| \\
& =|\beta|\left\|x+\beta^{k-1} P(x)\right\|=\|x+\omega P(x)\|>1+\|P\|-\varepsilon .
\end{aligned}
$$

Letting $\varepsilon \downarrow 0$, we get $\|\mathrm{Id}+P\| \geq 1+\|P\|$ and $P$ satisfies (DE).

(b) The above argument works in the real case if $k-1$ is odd.

As an immediate consequence, we obtain the following.

Corollary 3.3. Let $X$ be a Banach space and let $k$ be an integer, $k \geq 2$.

(a) If $X$ is complex, then the $k-D P$ and $k-A D P$ are equivalent.

(b) If $X$ is real and $k$ is even, then the $k-D P$ and $k$-ADP are equivalent.

Let us give more examples.

EXAMPLES 3.4.

(a) By Example 2.1(b), the complex space $\mathbb{C}$ has the $k$-ADP for every $k \in \mathbb{N}$. Then, by the above corollary, $\mathbb{C}$ has the $k$-DP when $k \geq 2$, but $\mathbb{C}$ does not have the 1-DP.

(b) Although there are non-homogeneous polynomials on $\mathbb{R}$ which do not satisfy (ADE) (see Example 2.1(c)), it is easy to check that the real space $\mathbb{R}$ has the $k$-ADP for every $k \in \mathbb{N}$.

(c) Thus, $\mathbb{R}$ has the $k-D P$ when $k$ is even by the above corollary. On the other hand, if $k$ is odd, $\mathbb{R}$ does not have the $k-D P$ as shown by the 
polynomial $P \in \mathcal{P}\left({ }^{k} \mathbb{R} ; \mathbb{R}\right)$ given by

$$
P(t)=-t^{k} \quad(t \in \mathbb{R}) .
$$

(d) By Corollary 2.10 and Example 2.11 we know that every polynomial from one of the complex spaces $c_{0}$, and $C(K), K$ arbitrary, into itself satisfies (ADE), but if we restrict ourselves to the case of $k$ homogeneous polynomials, Corollary 3.3 gives us more: The complex spaces $c_{0}$ and $C(K), K$ arbitrary, have the $k$-order Daugavet property for every $k \geq 2$.

REMARK 3.5. Item (c) in the above examples shows that, in the real case, the equations (DE) and (ADE) are not equivalent in $\mathcal{P}\left({ }^{k} X ; X\right)$ for odd $k$. It also shows that the $k$-DP and $k$-ADP are not equivalent in the real case for odd $k$.

The next results will show that the real spaces $c_{0}, c$ and $\ell_{\infty}$ do not have the $k$-ADP for any $k \geq 2$. We need some definitions. A closed subspace $Y$ of a Banach space $X$ is said to be an absolute summand of $X$ if there exists another closed subspace $Z$ such that $X=Y \oplus Z$ and, for every $y \in Y$ and $z \in Z$, the norm of $y+z$ only depends on $\|y\|$ and $\|z\|$. We then also say that $X$ is an absolute sum of $Y$ and $Z$. This implies that there exists an absolute norm $|\cdot|_{\text {a }}$ on $\mathbb{R}^{2}$ such that

$$
\|x+z\|=|(\|x\|,\|z\|)|_{\mathrm{a}} \quad(x \in X, z \in Z),
$$

where by an absolute norm we mean a norm $|\cdot|_{\mathrm{a}}$ on $\mathbb{R}^{2}$ such that $|(1,0)|_{\mathrm{a}}=$ $|(0,1)|_{\mathrm{a}}=1$ and $|(a, b)|_{\mathrm{a}}=|(|a|,|b|)|_{\mathrm{a}}$ for every $a, b \in \mathbb{R}$. We refer the reader to $[9, \S 21]$ for background. Examples of absolute summands are the $\ell_{p}$-sums of Banach spaces for $1 \leq p \leq \infty$.

The proof of the following result is straightforward. The reader may consult [11, Proposition 2.8] to see the main idea.

Proposition 3.6. Let $X$ be a Banach space and suppose that $X$ is an absolute sum of two closed subspaces $Y$ and $Z$. If $X$ has the $k$-DP (resp. $k$-ADP) for some $k \in \mathbb{N}$, then so do $Y$ and $Z$.

Now, we will study the relationship between the $k$-DP and $k$-ADP for different values of the integer $k$.

Proposition 3.7. Let $X$ be a Banach space and let $k$ be a positive integer. If $X$ has the $(k+1)-A D P$, then it has the $k-A D P$.

Proof. Fix $P \in \mathcal{P}\left({ }^{k} X ; X\right), P \neq 0$. For every $0<\varepsilon<\|P\|$, we may find $y \in S_{X}$ and $y^{*} \in S_{X^{*}}$ such that

$$
\|P(y)\|>\|P\|-\varepsilon \quad \text { and } \quad\left|y^{*}(y)\right|=1 .
$$


If we define $Q \in \mathcal{P}\left({ }^{k+1} X ; X\right)$ by

$$
Q(x)=y^{*}(x) P(x) \quad(x \in X),
$$

we have $\|Q\| \geq\|Q(y)\|=\left|y^{*}(y)\right|\|P(y)\|>\|P\|-\varepsilon$. Since $X$ has the $(k+1)$ ADP, we may find $\omega_{1} \in \mathbb{T}$ and $z \in S_{X}$ such that

$$
1+\|P\|-\varepsilon<\left\|z+\omega_{1} Q(z)\right\|=\left\|z+y^{*}(z) \omega_{1} P(z)\right\| .
$$

It follows that $\left|y^{*}(z)\right|>1-\varepsilon /\|P\|$ and, taking $\omega=\left(y^{*}(z) /\left|y^{*}(z)\right|\right) \omega_{1} \in \mathbb{T}$, we have

$$
\begin{aligned}
\|\operatorname{Id}+\omega P\| & \geq\|z+\omega P(z)\|=\left\|z+\frac{y^{*}(z) \omega_{1} P(z)}{\left|y^{*}(z)\right|}\right\| \\
& \geq\left\|z+y^{*}(z) \omega_{1} P(z)\right\|-\left|1-\frac{1}{\left|y^{*}(z)\right|}\right|\|P(z)\| \\
& >1+\|P\|-\varepsilon-\frac{\varepsilon\|P\|}{\|P\|-\varepsilon} .
\end{aligned}
$$

Letting $\varepsilon \downarrow 0$, we get $\|\operatorname{Id}+\omega P\|=1+\|P\|$ and $X$ has the $k$-ADP.

In the complex case, Proposition 3.7 can be read in terms of the $k$ Daugavet property for $k \geq 2$ since, in this case, the $k$-ADP and $k$-DP are equivalent.

Corollary 3.8. Let $X$ be a complex Banach space and let $k$ be a positive integer, $k \geq 2$. If $X$ has the $(k+1)-D P$, then it has the $k$-DP.

REMARKS 3.9.

(a) The above result is not valid for $k=1$. Indeed, the complex space $c_{0}$ has the 2-DP, but it does not have the 1-DP.

(b) The above result is false in the real case, since the real space $\mathbb{R}$ has the $2 m$-DP for every $m \in \mathbb{N}$, but it does not have the $(2 m-1)$-DP for any $m \in \mathbb{N}$.

In the real case a result similar to the above corollary can be proved if we allow a two-step jump.

Proposition 3.10. Let $X$ be a real Banach space and let $k$ be a positive integer. If $X$ has the $(k+2)-D P$, then it has the $k$-DP.

Proof. Fix $P \in \mathcal{P}\left({ }^{k} X ; X\right)$. For every $0<\varepsilon<1$, we may find $y \in S_{X}$ and $y^{*} \in S_{X^{*}}$ such that

$$
\|P(y)\|>\|P\|-\varepsilon \quad \text { and } \quad y^{*}(y)=1 .
$$

If we define $Q \in \mathcal{P}\left({ }^{k+2} X ; X\right)$ by

$$
Q(x)=\left(y^{*}(x)\right)^{2} P(x) \quad(x \in X),
$$


we have

$$
\|Q\| \geq\|Q(y)\|=\left(y^{*}(y)\right)^{2}\|P(y)\|>\|P\|-\varepsilon .
$$

Since $X$ has the $(k+2)$-DP, we may find $z \in S_{X}$ such that

$$
1+\|P(z)\|-\varepsilon \leq 1+\|P\|-\varepsilon<\|z+Q(z)\|=\left\|z+\left(y^{*}(z)\right)^{2} P(z)\right\| .
$$

It follows that $\left(y^{*}(z)\right)^{2}\|P(z)\|>\|P(z)\|-\varepsilon$, and so

$$
\begin{aligned}
\|\mathrm{Id}+P\| & \geq\|z+P(z)\|=\left\|z+\left(y^{*}(z)\right)^{2} P(z)\right\|-\left(1-\left(y^{*}(z)\right)^{2}\right)\|P(z)\| \\
& >1+\|P\|-2 \varepsilon .
\end{aligned}
$$

REMARK 3.11. The above result is not valid in the complex case for $k=1$. Indeed, the complex space $c_{0}$ has the 3 -DP but it does not have the $1-\mathrm{DP}$.

In $[12$, p. 141] there is given a weakly compact 2-homogeneous polynomial $P \in \mathcal{P}\left({ }^{2} \ell_{1} ; \ell_{1}\right)$ (both in the real and complex cases) which satisfies $v(P) \leq \frac{1}{2}\|P\|$. This fact, together with Propositions 1.3 and 3.7 , leads to the following example.

EXAMPLE 3.12. The space $\ell_{1}$ does not have the $k$-ADP for any $k \geq 2$.

REMARK 3.13. The converse of Proposition 3.7 is not true. For instance, $\ell_{1}$ has the $1-\mathrm{ADP}$ but it does not have the 2 -ADP.

It is actually proved in $[12$, p. 141$]$ that the polynomial $P: \ell_{1}^{2} \rightarrow \ell_{1}^{2}$ defined by

$$
P\left(x_{1}, x_{2}\right)=\left(\frac{1}{2} x_{1}^{2}+2 x_{1} x_{2},-\frac{1}{2} x_{2}^{2}-x_{1} x_{2}\right)
$$

satisfies $\|P\|=1$ and $v(P)=1 / 2$. Therefore, $\ell_{1}^{2}$ does not have the 2 -ADP. Since, in the real case, $\ell_{1}^{2}$ and $\ell_{\infty}^{2}$ are isometric, we infer that this latter space does not have the 2-ADP but, actually, much more examples can be deduced from this fact.

EXAMPLE 3.14. The real spaces $c_{0}, c, \ell_{\infty}, \ell_{\infty}^{n}$ for every $n \geq 2$ do not have the $k$-ADP for any $k \geq 2$. Indeed, since the real space $\ell_{\infty}^{2}$ does not have the 2 -ADP, and all the above spaces contain $\ell_{\infty}^{2}$ as an $\ell_{\infty}$-summand, Proposition 3.6 gives us the case $k=2$, and the rest follows from Proposition 3.7.

The above argument also shows that real $C(K)$ spaces when $K$ is a compact set with at least two isolated points do not have the $k$-ADP for any $k \geq 2$. But this assertion can be improved, as the next example shows.

EXAMPLE 3.15. Let $K$ be a non-perfect compact Hausdorff space with at least two points. Then the real space $C(K)$ does not satisfy the $k$-ADP for 
any $k \geq 2$. Indeed, we consider an isolated point $x_{1}$ of $K$ and $x_{2} \in K \backslash\left\{x_{1}\right\}$, and we define $p: C(K) \rightarrow \mathbb{R}$ by

$$
p(f)=f\left(x_{2}\right)^{2}-\frac{1}{2} f\left(x_{1}\right)^{2}
$$

for all $f \in C(K)$. Clearly $\|p\|=1=p\left(\chi_{K \backslash\left\{x_{1}\right\}}\right)$. Suppose that $C(K)$ has the 2 -ADP. Then, by Corollary 1.2 , we can find a sequence $\left(f_{n}\right)$ of norm-one elements of $C(K)$ such that

$$
\left|f_{n}\left(x_{2}\right)^{2}-\frac{1}{2} f_{n}\left(x_{1}\right)^{2}\right| \rightarrow 1 \text { and }\left\|\chi_{\left\{x_{1}\right\}}+f_{n}\right\| \rightarrow 2 .
$$

On the one hand, the first convergence implies that

$$
\left|f_{n}\left(x_{2}\right)\right| \rightarrow 1 \text { and }\left|f_{n}\left(x_{1}\right)\right| \rightarrow 0 .
$$

On the other hand, $\left\|\chi_{\left\{x_{1}\right\}}+f_{n}\right\|>1$ is only possible if

$$
\left\|\chi_{\left\{x_{1}\right\}}+f_{n}\right\|=\left|1+f_{n}\left(x_{1}\right)\right|
$$

and, therefore, the second convergence in (9) implies that

$$
\left|1+f_{n}\left(x_{1}\right)\right| \rightarrow 2,
$$

a contradiction.

Taking a look at all the examples that we have given, one may wonder if the $k$-DP or even the $k$-ADP for a Banach space $X$ implies that every $k$-homogeneous polynomial satisfies (ADE). This is not the case, as the following example shows.

Example 3.16. Consider the Banach space $C\left([0,1], \ell_{2}\right)$ and let $k$ be a positive integer. On the one hand, every weakly compact polynomial on $C\left([0,1], \ell_{2}\right)$ satisfies (DE) by Corollary 2.5. On the other hand, [11, Propositions 2.8 and 2.9] (see also the remark after Proposition 2.9 in [11]) shows that there exists a (non-weakly compact) $k$-homogeneous polynomial on $C\left([0,1], \ell_{2}\right)$ which does not even satisfy (ADE).

Acknowledgements. The authors would like to thank the referee, whose comments have improved the final form of the paper; in particular, for providing a shorter proof of Theorem 2.4.

\section{References}

[1] Y. Abramovich and C. Aliprantis, An Invitation to Operator Theory, Grad. Texts in Math. 50, Amer. Math. Soc., Providence, RI, 2002.

[2] -, - , Problems in Operator Theory, Grad. Texts in Math. 51, Amer. Math. Soc., Providence, RI, 2002.

[3] Y. A. Abramovich, C. D. Aliprantis, and O. Burkinshaw, The Daugavet equation in uniformly convex Banach spaces, J. Funct. Anal. 97 (1991), 215-230. 
[4] M. D. Acosta, Boundaries for spaces of holomorphic functions on $C(K)$, Publ. Res. Inst. Math. Sci. 42 (2006), 27-44.

[5] F. L. Bauer, On the field of values subordinate to a norm, Numer. Math. 4 (1962), 103-111.

[6] W. Bogdanowicz, On the weak continuity of the polynomial functionals defined on the space $c_{0}$, Bull. Acad. Polon. Sci. Cl. III 5 (1957), 243-246 (in Russian).

[7] F. F. Bonsall, B. E. Cain and H. Schneider, The numerical range of a continuous mapping of a normed space, Aequationes Math. 2 (1968), 86-93.

[8] F. F. Bonsall and J. Duncan, Numerical Ranges of Operators on Normed Spaces and of Elements of Normed Algebras, London Math. Soc. Lecture Note Ser. 2, Cambridge Univ. Press, 1971.

[9] - - - Numerical Ranges II, London Math. Soc. Lecture Note Ser. 10, Cambridge Univ. Press, 1973.

[10] K. Boyko, V. Kadets, M. Martín, and D. Werner, Numerical index of Banach spaces and duality, Math. Proc. Cambridge Philos. Soc., to appear.

[11] Y. S. Choi, D. García, S. G. Kim, and M. Maestre, The polynomial numerical index of a Banach space, Proc. Edinburgh Math. Soc. 49 (2006), 39-52.

[12] Y. S. Choi and S. G. Kim, Norm or numerical radius attaining multilinear mappings and polynomials, J. London Math. Soc. 54 (1996), 135-147.

[13] I. K. Daugavet, On a property of completely continuous operators in the space $C$, Uspekhi Mat. Nauk 18 (1963), no. 5, 157-158 (in Russian).

[14] J. Diestel and J. J. Uhl, Vector Measures, Math. Surveys 15, Amer. Math. Soc., Providence, 1977.

[15] S. Dineen, Complex Analysis on Infinite Dimensional Spaces, Springer Monogr. Math., Springer, London, 1999.

[16] J. Duncan, C. M. McGregor, J. D. Pryce, and A. J. White, The numerical index of a normed space, J. London Math. Soc. 2 (1970), 481-488.

[17] L. A. Harris, The numerical range of holomorphic functions in Banach spaces, Amer. J. Math. 93 (1971), 1005-1019.

[18] - , The numerical range of functions and best approximation, Proc. Cambridge Philos. Soc. 76 (1974), 133-141.

[19] V. M. Kadets and M. M. Popov, The Daugavet property for narrow operators in rich subspaces of $C[0,1]$ and $L_{1}[0,1]$, St. Petersburg Math. J. 8 (1997), 571584 .

[20] V. M. Kadets, R. V. Shvidkoy, G. G. Sirotkin, and D. Werner, Banach spaces with the Daugavet property, Trans. Amer. Math. Soc. 352 (2000), 855-873.

[21] M. Martín, The alternative Daugavet property of $C^{*}$-algebras and JB*-triples, Math. Nachr., to appear.

[22] M. Martín and T. Oikhberg, The alternative Daugavet property, J. Math. Anal. Appl. 294 (2004), 158-180.

[23] A. Rodríguez Palacios, Numerical ranges of uniformly continuous functions on the unit sphere of a Banach space, ibid. 297 (2004), 472-476.

[24] O. Toeplitz, Das algebraische Analogon zu einem Satze von Fejér, Math. Z. 2 (1918), 187-197.

[25] D. Werner, An elementary approach to the Daugavet equation, in: Interaction between Functional Analysis, Harmonic Analysis and Probability, N. Kalton et al. (eds.), Lecture Notes in Pure and Appl. Math. 175, Dekker, 1994, 449-454. 
[26] D. Werner, Recent progress on the Daugavet property, Irish Math. Soc. Bull. 46 (2001), 77-97.

Department of Mathematics

POSTECH

Pohang 790-784, Korea

E-mail: mathchoi@postech.ac.kr

Departamento de Análisis Matemático

Facultad de Ciencias

Universidad de Granada

18071 Granada, Spain

E-mail: mmartins@ugr.es
Departamento de Análisis Matemático

Universidad de Valencia

Doctor Moliner 50

46100 Burjasot (Valencia), Spain

E-mail: domingo.garcia@uv.es

manuel.maestre@uv.es

Received March 24, 2006

Revised version September 13, 2006 